\title{
Brain Tumor Management Amidst COVID-19 Pandemic
}

\author{
Kunal Kishor ${ }^{1}$, Dinesh Kumar Sinha1, Manish Kumar², Shashi Singh Pawar', \\ Richa Madhawi', Shraddha Raj', Rajesh Kumar Singh', Seema Devi', Rajiv \\ Ranjan Prasad ${ }^{1}$
}

${ }^{1}$ Department of Radiation oncology, State Cancer Institute, Indira Gandhi Institute of Medical Sciences, Patna, India. ${ }^{2}$ Department of Surgical oncology, State Cancer Institute, Indira Gandhi Institute of Medical Sciences, Patna, India.

\begin{abstract}
In May, Brain Tumor Awareness Month, we are facing challenges pertaining to brain cancer and coronavirus (COVID-19) pandemic. With no effective treatment and no vaccine currently available, the most effective means of slowing the dissemination of COVID-19 is through social distancing, crowd avoidance, appropriate quarantine, and other techniques intended to limit community exposure. However, in this ongoing pandemic era, timely treatment by surgery is critical to reduce the tumor mass inside a patient's brain. By giving drug therapy to slow down the tumor's progression and provide a longer survival time is utmost important. As hospitals across the country postpone elective procedures to increase the capacity for COVID-19 patients, there is a lot of uncertainty among the neuro-oncology community. Patient having multiple co-morbidities poses challenges for centers that may need access to ventilator care due to insufficient critical care facilities across the country. This crisis is straining our health care system, as this viral infection continues to escalate. It is the time for the neuro-oncology team to develop a uniform and acceptable approach to the brain tumor patient. We present an approach developed by an international multidisciplinary group to provide them best possible care during this pandemic as well as reducing their chance of viral infection.
\end{abstract}

Keywords: Brain tumor- covid-19- pandemic

Asian Pac J Cancer Care, 5 (Suppl 1), 235-238

\section{Introduction}

Corona viruses are a large family of viruses causing respiratory infections ranging from the common cold to more severe diseases such as Middle East Respiratory Syndrome (MERS) and Severe Acute Respiratory Syndrome (SARS). The most recently discovered coronavirus causes coronavirus disease COVID-19. This disease was unknown before the outbreak began in Wuhan, China, in December 2019 [1]. WHO has declared 2019 novel coronavirus disease (COVID-19), caused by SARS-CoV-2, a public health emergency of international concern. Reports from China and many other countries show that cancer patients and cancer survivors are at higher risk to get infected with the novel coronavirus and more likely to die from complication of COVID [2]. COVID-19 pandemic is an unprecedented challenge and is rapidly evolving which result in de-prioritisaton of essential medical care.
Submission Date: 07/17/2020Ａcceptance Date: 08/15/2020

\footnotetext{
Corresponding Author:

Dr. Kunal kishor

Department of Radiation oncology, State Cancer Institute, Indira Gandhi Institute of Medical Sciences, Patna, India.

Email:kkishor28@gmail.com
}

\section{Initial preparation}

American society of clinical oncology (ASCO) encourages existing Centre for Disease Control and prevention (CDC) guidelines in the form of general health care facility and health care professional guidance, clinical care guidance, home care guidance, high-risk subpopulation guidance [3].

As per WHO guidelines, hand washing and social distancing are recommended. Frequent hand washing for at least 20 seconds is important or using a $60 \%$ alcohol hand sanitizer - gloves are not a substitute. Do not shake hands and avoid touching your face. Multiple facilities are recommending a limit of one adult accompanying a patient. The most important goal is to reduce the risk of transmission of COVID-19 and to allow cancer care to continue for those most likely to benefit [4]. 
ANTI-CANCER THERAPY FOR PATIENTS WITH COVID-19 INFECTION: Should cancer therapy be delayed in patients who are infected with COVID-19?

Interrupting anti-cancer treatment in patients with active COVID-19 should be strongly considered as continuation of treatment may lead to further immune suppression and risk for serious complications [5]. The United Kingdom National Institute for Health and Care Excellence (NICE) has published rapid guidance on the delivery of anti-cancer therapy that suggests treatment may be initiated or resumed after one negative SARS-Cov-2 test.

SURGERY: Can/should surgery be cancelled or delayed?

The American College of Surgeons (ACS) has issued guidance and provides additional advice related to triage of patients for surgery relevant to cancer care. However, clinicians and patients will need to make individual determinations based on the potential harms of delaying needed cancer-related surgery; in many cases these surgeries cannot be considered "elective". Also, if the surgery requires post-operative intensive care, the current capacity of the intensive care units available for that care should be considered as part of decision making.

The Society of Surgical Oncology (SSO) has released brief guidance on surgery for different tumor types. The risks of tumor progression with delay in definitive surgery should be weighed against the potential added burden on hospital resources, case complexity and patient risk of exposure to COVID-19.

National Foundation for Cancer Research's Chief strategy officer, Michael Wang shared his advices for cancer patients, survivors and caregivers during the COVID-19 pandemic [6].

1. A comprehensive evaluation should be performed before starting treatment, based on patient's clinical symptoms, tumor images, molecular testing results, and planned surgery scale and location.

2. In newly diagnosed neuroglioma, if neurological symptoms are limited and stable, imaging shows only a low grade tumor and the tumor could be removed completely even at a later time, postponed surgery schedule is acceptable if the patients could be closely monitored.

3. In case of a high grade tumor, including GBM, if the patient is young and in good general condition, then maximum resection of tumor should be performed without delay, so long as best practices to prevent coronavirus infection are in place.

4. For elderly patients with a poor general condition and associated co-morbidities, biopsy procedure or surgery are not suggested. Instead, chemotherapy should be planned according to the patient's conditions. When a clear diagnosis can't be concluded because of limited resources in hand, then follow-up MRI procedures are suggested every three to six months in order to evaluate the speed of tumor progression. Then treatment plan should be developed for the patient accordingly. Until unless a clear picture of tumor's feature is not obtained, no aggressive therapy should be given to elderly patients.
5. Tumor's biomarker test is critical for treatment plan selection. If a patient with recurrent GBM has the biomarker called "wild type IDH," surgery should be performed quickly - as this type of tumor usually grows rapidly and causes harmful neurological symptoms. If a recurrent GBM patient has the biomarker called "MGMT positive," surgery could be postponed under close monitoring procedures, as this type of GBM is more controllable by chemotherapy drugs such as Temozolomide (TMZ).

6. If a patient is on tumor treating fields (TTF) treatment, the procedure should be performed remotely, such as at home, and appropriate training should be provided to patients or their family members so as to reduce the risk of being unnecessarily exposed to coronavirus in hospital settings.

\section{ESMO has adapted following neurosurgery protocol}

\section{Priorities for brain tumour patients: Neurosurgery}

High Priority

- Need for acute decompression

- Maximal safe resection in suspected malignant glioma

- Diagnostic biopsy in suspected primary central nervous system lymphoma

\section{Medium Priority}

- Resection/biopsy of non-contrast enhancing primary brain tumour with stable neurological symptoms

- Re-resection of recurrent lower WHO grade glioma

\section{Low Priority}

- Partial resection of recurrent malignant glioma

RADIATION: Can/should the initiation of radiation be delayed? Can radiation be interrupted or postponed if already in progress?

ASCO encourages clinicians to follow ASTRO's current guidance. ASTRO recommends hypofractionated schedules as reasonable. ASCO recognizes the risks of delay in treatment for patients with rapidly progressing, potentially curable tumors may outweigh the risks of COVID-19 exposure/infection, but patients receiving radiation for symptom control or at low risk of harm due to alteration of schedule for radiation treatment visits could potentially be safely delayed. Patients should check with their radiation oncologist to determine the most appropriate course of action for their treatment. NICE has published rapid guidance on the delivery of radiation which may be of value [7].

ESMO has made following radiotherapy protocol [8]:

\section{Priorities for brain tumour patients: Radiotherapy}

\section{High Priority}

- Radiotherapy of newly diagnosed glioblastoma, IDH wild type

- Radiotherapy of lower WHO grade gliomas, IDH 
mutant with relevant clinical manifestations

- Radiotherapy of adult medulloblastoma

\section{Medium Priority}

- Radiotherapy of lower WHO grade gliomas, IDH mutant

\section{Low Priority \\ - Re-irradiation of gliomas}

IMMUNOSUPPRESSIVE THERAPY: Can/should potentially immunosuppressive therapy be stopped, delayed, or interrupted?

There is no little direct evidence to guide decisions around changing or withholding immunosuppressive therapy in patients with cancer. Therefore, routinely withholding critical anti-cancer or immunosuppressive therapy is not recommended. The balance of potential harms that may result from delaying or interrupting treatment versus the potential benefits of possibly preventing or delaying COVID-19 infection is very uncertain. Clinical decisions should be individualized and consider factors such as the risk of cancer recurrence/ progression if therapy is delayed, modified or interrupted; the number of cycles of therapy already completed; and the patient's tolerance of treatment [3-9].

\section{ESMO has made following practice points [8]}

Priorities for brain tumour patients: Systemic therapy

\section{High Priority}

- High-dose chemotherapy (with methotrexate) for newly diagnosed primary central nervous system lymphoma (PCNSL)

- Temozolomide concurrent with and adjuvant to radiotherapy for newly diagnosed glioblastoma with MGMT promoter methylation

- Temozolomide after radiotherapy for IDH-mutant 1p19q-intact anaplastic astrocytoma

-Alkylating chemotherapy after radiotherapy in newly diagnosed 1p19q-codeleted anaplastic oligodendroglioma

- Alkylating chemotherapy for recurrent glioma with MGMT promoter methylation

- Strict control of steroid prescription ("as little as possible, as much as needed")

\section{Medium Priority}

- Temozolomide concurrent with and adjuvant to radiotherapy for newly diagnosed glioblastoma without MGMT promoter methylation

- Systemic therapy for progressive brain tumours without evidence, e.g. meningioma or ependymoma in adults

- Alkylating chemotherapy after radiotherapy in IDH-mutant WHO grade II astrocytoma

- Adjuvant chemotherapy after radiotherapy for adult medulloblastoma

\section{Low Priority}

- Alkylating chemotherapy in patients with recurrent gliomas lacking MGMT promoter methylation, patients with 2nd or higher recurrence of glioma, and patients with reduced performance status or in advanced age.

\section{Looking Forward}

The Covid-19 pandemic has presented an uncertain situation for health care facilities including cancer care, and we have to optimize ourselves according to daily updates in covid-19 related research and knowledge, for better management of cancer patients, who are in utmost need of treatment. But, the future is unpredictable as nobody knows, how long the pandemic will remain and moreover anxiety of second and third waves. Therefore, we have to be prepared among frequently changing policies and recommendations. With experiences of previous pandemics, we expect that we will be out of this unprecedented time some day or later, but we have to get through the financial and emotional impacts. So, whatever we are facing challenges at present times, we have to maneuvers these, to deliver judicious and better patient care to be proud of, what we did for our society in most critical times.

\section{Conflict of Interest}

No authors have any conflict of interest to disclose.

Financial Disclosure

None declared 


\section{References}

1. www.mohfw.gov.in

2. Liang W, Guan W, Chen R, Wang W, Li J, Xu K, Li C, Ai Q, Lu W, Liang H, Li S, He J. Cancer patients in SARSCoV-2 infection: a nationwide analysis in China. The Lancet Oncology. 2020 03;21(3):335-337. https://doi.org/10.1016/ s1470-2045(20)30096-6

3. www.ASCO: COVID19 Patient care information..

4. www.ASTRO:COVID19 Recommendation and informationAmerican Society for Radiation Oncology (ASTRO).

5. Zhang L, Zhu F, Xie L, Wang C, Wang J, Chen R, Jia P, Guan H, Peng L, Chen Y, Peng P, Zhang P, Chu Q, Shen Q, Wang Y, Xu S, Zhao J, Zhou M. Clinical characteristics of COVID-19-infected cancer patients: a retrospective case study in three hospitals within Wuhan, China. Annals of Oncology. 2020 07;31(7):894-901. https://doi.org/10.1016/j. annonc.2020.03.296

6. Nfcr.org/To Do the Surgery or Not in the Midst of COVID-19 Pandemic: "New Normal" Treatment Guidelines for Brain Tumor Patients.

7. www.nice.org.uk/guidance/ng161/prioritizing-systemicanticancer-treatments.

8. ESMO: Brain tumor in COVID-19 era.

9. Russell B, Moss C, George G, Santaolalla A, Cope A, Papa S, Van Hemelrijck M. Associations between immune-suppressive and stimulating drugs and novel COVID-19-a systematic review of current evidence. ecancermedicalscience. 202003 27;14. https://doi. org/10.3332/ecancer.2020.1022

\section{Q $\odot$}

This work is licensed under a Creative Commons AttributionNon Commercial 4.0 International License. 